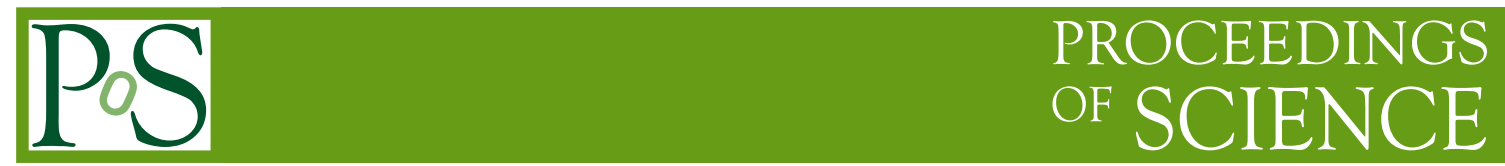

\title{
Optimisation of a beta beam
}

\section{ANDREA DONINI*}

Instituto de Fisica Teorica (UAM/CSIC)

E-mail: andrea.donini@uam.es

\section{MATS LINDROOS}

CERN

E-mail: mats.lindroos@mail.cern.ech

In this talk we have tried to shortly address some of the problems related with the optimization of those $\beta$-beam facilities that can be developed using existing or projected infrastructures at CERN (such as the PS, the SPS, the PS2 and the SPS+). The relative impact of an increase in the number of stored ions or of the boosting factor $\gamma$ on the sensitivity to $\theta_{13}, \delta$ or the sign of the atmospheric mass difference is reviewed, together with issues related to the production, acceleration and storage of ${ }^{6} \mathrm{He},{ }^{18} \mathrm{Ne},{ }^{8} \mathrm{Li}$ and ${ }^{8} \mathrm{~B}$ ions.

10th International Workshop on Neutrino Factories, Super beams and Beta beams June 30 - July 52008

Valencia, Spain

\footnotetext{
* Speaker.
} 
The full understanding of the leptonic mixing matrix constitutes, together with the discrimination of the Dirac/Majorana character of neutrinos and with the measurement of their absolute mass scale, the main neutrino-physics goal for the next decade. New experiments have been proposed to look for the intimately related parameters $\theta_{13}$ and $\delta$ through "appearance channels" such as $v_{e} \leftrightarrow v_{\mu}$ (the "golden channel") and $v_{e} \rightarrow v_{\tau}$ (the "silver channel") [1]. Strong correlations between $\theta_{13}$ and $\delta$ [2], and the presence of parametric degeneracies in the $\left(\theta_{13}, \delta\right)$ parameter space [3], make the simultaneous measurement of the two variables extremely difficult. Most of the proposed solutions to these problems imply the combination of different experiments and facilities, such as reactors (Double Chooz [4] should start data taking in 2008), Super-Beams (of which T2K [5] is the first approved one), $\beta$-Beams [6] or the Neutrino Factory [7].

A list of observables to be measured has been defined to compare different options: $\theta_{13}$; the $\mathrm{CP}$-violating phase $\delta$; the sign of the atmospheric mass difference; the deviation from $\theta_{23}=45^{\circ}$; the $\theta_{23}$-octant (if $\theta_{23} \neq 45^{\circ}$ ). Aside from these measurements, a new facility should also reduce the present errors on atmospheric and solar parameters. A comparison of all the proposed facilities based on this "shopping list" has been presented in the International Scoping Study of a future Neutrino Factory and SuperBeam facility (ISS) Report [8]. The outcome of this comparison is that the "ultimate" neutrino oscillation experiment is a Neutrino Factory with $20-30 \mathrm{GeV}$ stored muons, whose (anti)neutrino fluxes aim at two 50 Kton magnetized iron detectors located at $L \in$ [2000,4000] Km and $L \sim 7000 \mathrm{Km}$ from the source, respectively. The goal luminosity for such facility is $5 \times 10^{20}$ useful muon decays per year per polarity per baseline.

The nearest competitor of this setup is the "high $\gamma^{6} \mathrm{He} /{ }^{18} \mathrm{Ne} \beta$-Beam", proposed in Refs. [9, 10], in which ${ }^{6} \mathrm{He}$ and ${ }^{18} \mathrm{Ne}$ ions are boosted at $\gamma \in[300,600]$ and injected into a storage ring aiming at a 1 Mton water Čerenkov detector located at $L=650 \mathrm{Km}$ from the source, with a nominal luminosity of $2.9 \times 10^{18}{ }^{6} \mathrm{He}\left(1.1 \times 10^{18}{ }^{18} \mathrm{Ne}\right)$ useful ion decays per year. Other proposals based on the $\beta$-beam technology have been advanced in recent times, using ${ }^{8} \mathrm{Li}$ and ${ }^{8} \mathrm{~B}$ ions as neutrino emitters [11]. These two ions, if produced with a sufficient rate [12, 13], give neutrino fluxes of energies higher than those that can be attained using ${ }^{6} \mathrm{He}$ and ${ }^{18} \mathrm{Ne}$. Detectors located at the socalled "magic baseline" [14] illuminated by a $\beta$-beam can thus be envisaged [15], with a very high sensitivity to the neutrino mass hierarchy. Combination of one far detector with a near one (at the oscillation peak) is an interesting possibility that has also been advanced [16, 17].

Optimization of the $\beta$-beam technology should now be pursued, as it was the case for the Neutrino Factory. In this talk, we have tried to address some of the most important problems we face to maximize the physics reach of neutrino oscillation detectors exposed to a $\beta$-Beam flux. We restrict ourselves to three $\beta$-Beam setups for which only CERN-based infrastructures (the PS, the SPS and their proposed upgrades in the framework of the LHC maintainance and upgrade programme, the PS2 and the SPS+) are used to accelerate ions to the desired $\gamma$ : (a) Low- $\gamma \beta$-beam with ${ }^{6} \mathrm{He} /{ }^{18} \mathrm{Ne}$; (b) High- $\gamma \beta$-beam with ${ }^{6} \mathrm{He} /{ }^{18} \mathrm{Ne}$; (c) High- $\gamma \beta$-beam with ${ }^{8} \mathrm{Li} /{ }^{8} \mathrm{~B}$. The problems discussed in this session affect mainly these three setups. Other proposals have been suggested, such as very low $\gamma \beta$-beams (see, e.g., Ref. [19] and refs. therein), relevant for nuclear physics studies, or very high $\gamma \beta$-beams (see Refs. $[9,20]$ ). Issues specific to these setups will not be covered here. 


\section{Questions}

To address some of the relevant questions, it is useful to remind two straighforward scaling laws that relate the boosting factor $\gamma$, the electron end-point energy $E_{0}$ and the total number of ions that decay in the storage ring aiming at the far detector, $N_{\beta}$. First of all, the physics that can be studied at a given detector depends on the neutrino energy in the lab rest frame, $E$, whose maximum value is $E_{\max }=2 \gamma E_{0}$. We can therefore study the same physics (i.e., we have the same neutrino flux) changing $\gamma$ at the same time we change ions according to the scaling law:

$$
\frac{\gamma^{H e / N e}}{\gamma^{L i / B}}=\frac{E_{0}^{L i / B}}{E_{0}^{H e / N e}}
$$

Secondly, the sensitivity to the specific physics observable that can be studied by using a given neutrino flux is strongly dependent on the number of events that can be detected. For a $\beta$-beam with $E>1 \mathrm{GeV}$ (for which the $v N$ cross-section increases linearly), we have:

$$
N_{\text {events }} \propto N_{\beta} \times E \times\left(\frac{\gamma^{2}}{L^{2}}\right) \times P_{e \mu}(L / E) \propto N_{\beta} \frac{\gamma}{E_{0}},
$$

where the last relation is obtained when the $L / E$ ratio is tuned at the first peak of the oscillation probability [6]. Eventually, using eq. (1.1), we get [18]:

$$
\frac{N_{\beta}^{L i / B}}{N_{\beta}^{H e / N e}}=\left(\frac{E_{0}^{L i / B}}{E_{0}^{H e / N e}}\right)^{2}
$$

Armed with these two scaling laws we can try to understand if it is better to increase $\gamma$ or the number of stored ions $N_{\beta}$ to reach a goal sensitivity to a given physics observable. In Fig. 1(top right) we see that at fixed ion (in this case, $\mathrm{He} / \mathrm{Ne}$ ) and $N_{\beta}$ increasing $\gamma$ makes a real difference when looking for a CP-violating signal. In particular we see that, for a $\mathrm{He} / \mathrm{Ne}$ beam aiming at a 1 Mton water Čerenkov detector located at the first $v_{e} \rightarrow v_{\mu}$ oscillation peak, raising $\gamma$ from $\gamma \sim 100$ to $\gamma=350$ the sensitivity to $\delta$ improves from $\sin ^{2} 2 \theta_{13} \sim 10^{-3}$ to $\sin ^{2} 2 \theta_{13} \sim 10^{-4}$. On the other hand, we see from Fig. 1(top left) that, for a He/Ne beam at $\gamma=350$, increasing $N_{\beta}$ from $2 \times 10^{18}$ to $2 \times 10^{19}$ useful decays per year the maximal sensitivity to $\theta_{13}$ only improves from $\sin ^{2} 2 \theta_{13} \sim 4 \times 10^{-5}$ to $\sin ^{2} 2 \theta_{13} \sim 2 \times 10^{-5}$. From these two plots we can see that, for $\mathrm{He} / \mathrm{Ne}$ beams, the increase in $\gamma$ is much more important than the increase in $N_{\beta}$, a consequence of the detector technology that can be used in this case, i.e. a 1 Mton water Čerenkov detector.

This is not the case for Li/B beams. In this case, the detector technology adopted is either magnetized iron detectors (MIND-style) or TASD, in both cases with a mass of tens of Ktons. From Fig. 1(bottom left) we see that, in this case, increasing $N_{\beta}$ from $2 \times 10^{18}$ to $2 \times 10^{19}$ useful decays per year the $\theta_{13}$-sensitivity improves from $\sin ^{2} 2 \theta_{13} \sim 9 \times 10^{-4}$ to $\sin ^{2} 2 \theta_{13} \sim 1.5 \times 10^{-4}$, i.e. almost an order of magnitude. Whilst $\mathrm{He} / \mathrm{Ne} \gamma=350$ beams are systematics-dominated, $\mathrm{Li} / \mathrm{B}$ beams at the same $\gamma$ are statistics-dominated. In this latter case, an increase in the number of stored ions can make a big difference. Notice that a possible bottleneck could be represented by the duty factor needed to maintain the atmospheric neutrino background at an acceptable level. From Fig. 1(bottom right) [21] we see that the expected atmospheric background goes down from 

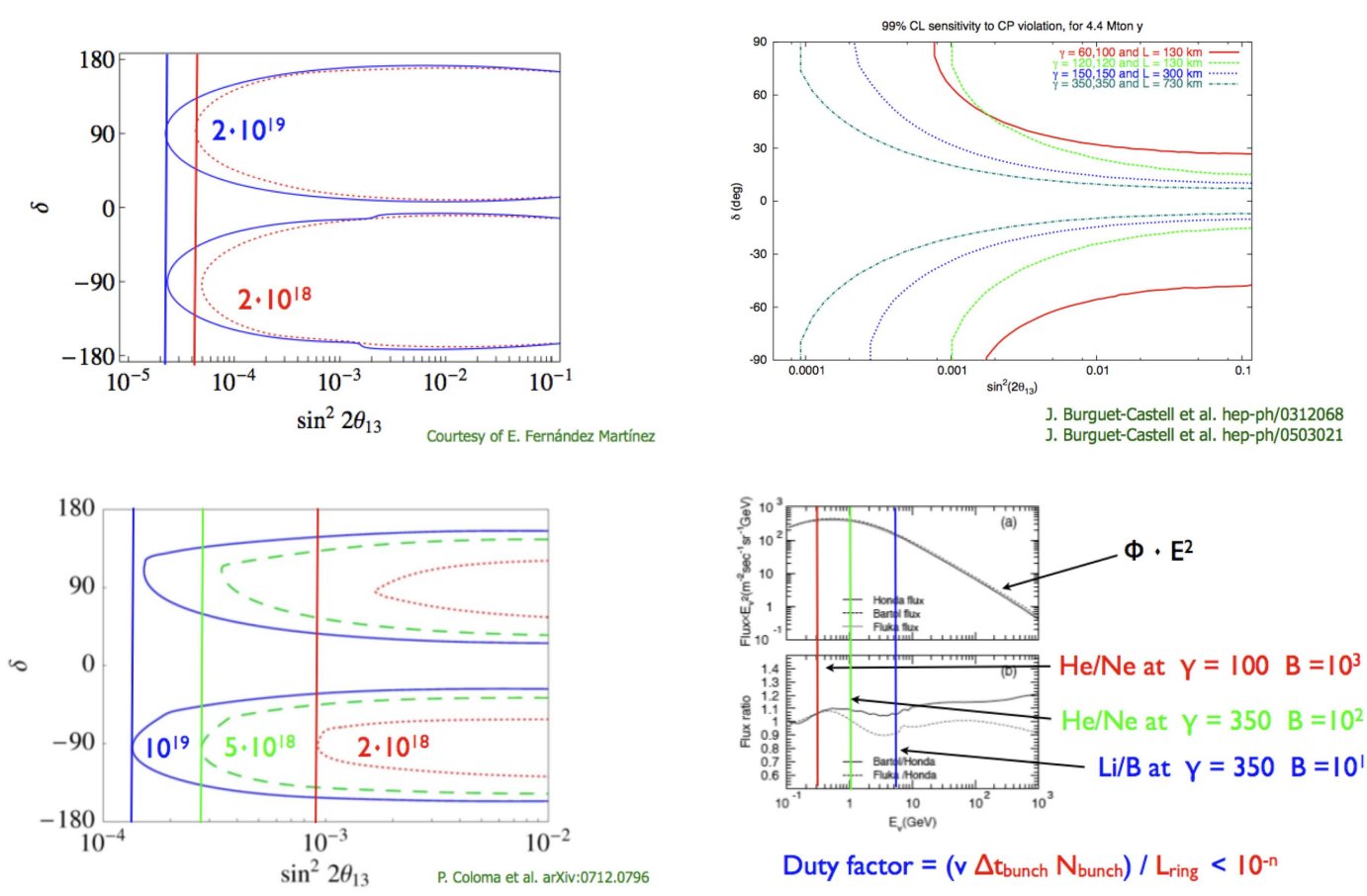

Duty factor $=\left(v \Delta t_{\text {bunch }} N_{\text {bunch }}\right) / L_{\text {ring }}<10^{-n}$

Figure 1: Top left: the impact of $N_{\beta}$ on $\theta_{13}$-sensitivity at high- $\gamma$ He/Ne beams; top right: the impact of $\gamma$ on the $\delta$-sensitivity at high- $\gamma$ He/Ne beams; bottom left: the impact of $N_{\beta}$ on $\theta_{13}$-sensitivity at high- $\gamma \mathrm{Li} / \mathrm{B}$ beams; bottom right: the atmospheric neutrino background for the three setups.

$\mathrm{O}(1000)$ events per year for $\gamma=100 \mathrm{He} / \mathrm{Ne}$ beams (i.e., neutrinos with an energy in the range $E \in[200,300] \mathrm{MeV})$ to $\mathrm{O}(10)$ events for $\gamma=350 \mathrm{Li} / \mathrm{B}$ beams. The duty factor can thus be correspondingly relaxed. This can ease the storage issues for this particular setup with respect to options with low $\gamma \mathrm{He} / \mathrm{Ne}$ setups.

A totally different issue is that of the activation of the different facilities used in the production, acceleration and storage stages of a $\beta$-beam facility. A preliminar study for the low $\gamma \mathrm{He} / \mathrm{Ne}$ setup has been presented at the NuFact' 07 workshop, showing that in principle for this facility activation problems can be overcome. A similar study for higher $\gamma$ options and for $\mathrm{Li} / \mathrm{B}$ setups is lacking, though. It seems, however, that the potentially dangerous stage is represented in CERN-based schemes by the PS (at which ions are boosted up to $\gamma \sim 10$ ). No specific problems should arise in the SPS or SPS+ acceleration stage (where ions are boosted up to the ultimate $\gamma$ ).

\section{Answers}

\section{1 introduction}

The first conceptual design work for a $\beta$-beam facility was done at CERN in 2002 [22] during a period when CERN faced immense financial challenges. As a result, the $\beta$-beam work came to focus on the potential re-use of a large part of the CERN accelerator complex where the R\&D need was limited and with a minimum of new constructions. The physics goal was set so that the facility would be better than any neutrino oscillation facility presently under construction. While this was successful in persuading CERN management and the European Nuclear Physics community that 
Table 1: Some possible decay ring options for a different Lorenz gamma of ${ }^{6} \mathrm{He}$. The decay ring arcs are for all cases considered to be completely filled with dipoles.

\begin{tabular}{llll}
\hline Gamma & $\begin{array}{l}\text { Rigidity } \\
{[\mathrm{Tm}]}\end{array}$ & Ring length $^{a}$ & Dipole Field $^{b}$ \\
\hline 100 & 935 & 4197 & 3.1 \\
150 & 1403 & 6296 & 4.7 \\
200 & 1870 & 8395 & 6.2 \\
350 & 3273 & 14691 & 10.9 \\
500 & 4676 & 20987 & 15.6 \\
\hline \multicolumn{4}{c}{ Assuming a fixed field of 5 T and a single } \\
straight section of 36\% of the circumference \\
${ }^{b}$ Assuming a arc radius of $300 \mathrm{~m}$ and a decay \\
\multicolumn{4}{c}{ ring length of $6885 \mathrm{~m}$}
\end{tabular}

a more ambitious design effort should be made for $\beta$-beams, it also left the neutrino community with the impression that this rather limited scope also represented the ultimate physics reach of the $\beta$-beam concept. The following sections are rather speculative and aims to demonstrate that the $\beta$-beam concept can be taken much further (budget permitting).

\subsection{Higher Lorenz $\gamma$}

The reason for limiting $\gamma$ to 150 for ${ }^{6} \mathrm{He}$ and 250 for ${ }^{18} \mathrm{Ne}$ in the first study [22] is the SPS at CERN. The SPS is the last accelerator in the CERN Large Hadron Collider injector chain and as it also could serve as the last accelerator for a possible $\beta$-beam facility at CERN it will set the limit for the top $\gamma$. The LHC is a collider with a very slow energy ramp and would be highly unsuitable for the acceleration of radioactive ions to higher energies. To reach higher $\gamma$ 's a new injector is needed and in a conceptual study [23] at CERN of possible injectors upgrades for future physics needs it has been named SPS+. This hypotetical machine should be able to reach at least twice the $\gamma$ of the existing SPS e.g. $\gamma=300$ for ${ }^{6} \mathrm{He}$ and $\gamma=500$ for ${ }^{18} \mathrm{Ne}$.

At these $\gamma$ 's the decay ring must either be a lot larger if the presently studied dipole are kept for arcs or a new design for dipoles with much larger magnetic field has to be done, see Table 1. Higher $\gamma$ values seems to have a clear advantage in that the back-ground problem in the detectors appears to be much less severe as the background at the $\gamma$ used in [22] necessitates a very small duty factor, i.e. some $10^{-3}$. Such a small duty factor requires machine physicists to come up with fancy accumulation schemes such as the asymmetric bunch merging scheme [24] used in [22]. This scheme allows the successive accumulation of many bunches within the same bucket in the decay ring but it is limited by the longitudinal phase space available in the decay ring. For duty factors $\geq 0.1$ it should be possible to use a barrier bucket scheme [25] where the ions are kept un-bunched between to RF barriers, injection is done next to the unbunched beam and injected particles are adiabatically merged with the unbunched ions using an additional "movable" RF barrier. Such a scheme would probably permit a large efficiency in the merging and would also reduce the peak current in the ring. The latter is important to reduce space charge problems in the decay ring. 


\section{$6 \mathrm{He}$}

Annual rate

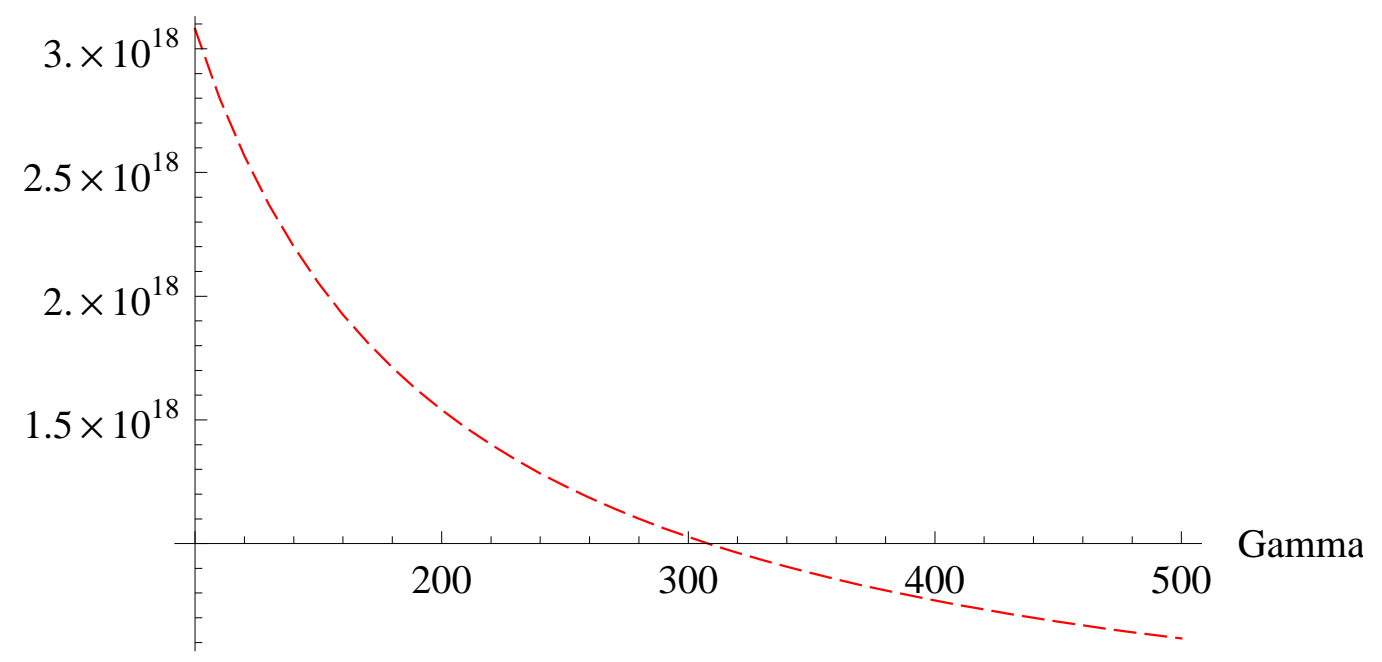

Figure 2: The annual rate as a function of the $\gamma$ in the decay ring for $10{ }^{14}{ }^{6} \mathrm{He}$ ions stored in the ring. One of the straight sections of the ring represent $36 \%$ of the total circumference.

\subsection{Higher intensities}

The intensity at a $\beta$-beam facility is set by the production rate and the efficiency of the acceleration and stacking scheme. The production of ${ }^{6} \mathrm{He}$ and ${ }^{18} \mathrm{Ne}$ in the first study at CERN [22] was proposed to be done using the ISOL method. In this method a thick and hot target is irradiated with a proton beam of a few GeV. Spallation, fragmentation and fission is induced in the target and a large number of isotopes will diffuse out of the hot target. The isotopes are guided to a ion source and are there after extracted and mass separated so that a pure beam of a single isotope can be delivered to the user. The method is well suited for the production of intense beams with good beam quality so it seemed the ideal choice for a $\beta$-beam facility. However, the methods as applied by Nuclear physicists are normally optimized for very exotic elements with high isospin which are of little interest for $\beta$-beams. A radioactive beam experiment using an exotic isotope can usually operate with a yield as low as $10^{4}-10^{5}$ exotic isotopes per second.

There are alternative productions scenarios which are all versions of the ISOL method but better adapted to the need of $\beta$-beams e.g. the neutron converter, direct production and production ring concepts. Several studies have been done for production of isotopes of interest for $\beta$-beam using these different concepts. In Table 2 estimates for the achievable productions rates are given and a short description of each concept is given below. The neutron converter concept has been proposed to be used at, e.g., the ZARAF facility in Israel for light ion production [27]. The target in which the radioactive ions are produced are only irradiated by neutrons which induces less heating and less radiation damage. The neutrons are produced through spallation in another cooled and robust target on which the primary protons impinge. Consequently, the flux of the primary proton beam can be higher and as many neutron induced production channels have a large cross section the intensity of the extracted ion beam will be more intense than for classical ISOL production. 
Table 2: Estimates made by the authors for the production rate per second in the target of a few isotopes of interest for $\beta$-beams. Note that the references refers to the method rather than to the production limits.

\begin{tabular}{llll}
\hline Isotope & Method & $\begin{array}{l}\text { Rate within reach } \\
\text { ions/second }\end{array}$ & Reference \\
\hline${ }^{18} \mathrm{Ne}$ & ISOL at $1 \mathrm{GeV}$ and $200 \mathrm{~kW}$ & $<8 \times 10^{11}$ & {$[22]$} \\
${ }^{6} \mathrm{He}$ & ISOL converter at $1 \mathrm{GeV}$ and $200 \mathrm{~kW}$ & $<5 \times 10^{13}$ & {$[22]$} \\
${ }^{18} \mathrm{Ne}$ & Direct production through ${ }^{16} \mathrm{O}\left({ }^{3} \mathrm{He}, \mathrm{n}\right)^{18} \mathrm{Ne}$ & $<1 \times 10^{13}$ & {$[26]$} \\
${ }^{6} \mathrm{He}$ & ISOL converter at $40 \mathrm{MeV}$ Deuterons and $80 \mathrm{~kW}$ & $<6 \times 10^{13}$ & {$[27]$} \\
${ }^{8} \mathrm{Li}$ & Production ring through ${ }^{7} \mathrm{Li}(\mathrm{d}, \mathrm{p})^{8} \mathrm{Li}$ & $<1 \times 10^{14}$ & {$[12]$} \\
\hline
\end{tabular}

The direct production method [26] is the classical method of secondary ion production in nuclear physics. Two nuclei are merged at energies just above the couloumb barrier using an accelerated light ion beam impinging on a target. The cross sections for these type of reaction are large and as long as suitable projectiles and targets can be found the ratio of produced secondary ions versa impinging ions is excellent.

Recently it has been proposed to enhance direct production with the help of a storage ring $[12,13]$. The projectile ions are circulating in the ring with a thin target in the ring orbit. The circulating ion beam will effectively be cooled by the combination of ionization losses in the target and repeated re-accelerating in a RF cavity in the ring. If the target is positioned in a dispersive region [12] and wedge shaped the beam is also longitudinally cooled. The beam energy has to be tuned to the optimum energy for the desired reaction channel but it also has to be kept high enough to assure that the beam is fully stripped after the target. The secondary ions are proposed [12] to be collected with foils surrounding the beam after the target.

The magnet protection system will be increasingly challenging for higher intensities [28] and it is probably necessary to use an open mid-plane superconducting dipole design to manage the losses in the decay ring arches, [29]. The accumulation scheme proposed for the facility described in [22] requires collimation of the particles pushed out of the RF bucket and at higher intensities these risks become unmanageable. The use of the earlier proposed barrier bucket scheme for accumulation should overcome this problem and should - if efficient - result in a larger flux for the price of much large duty factor $(>0.1)$. The loss of potential flux due to the inherent limits of the asymmetric bunch merging scheme [24] used in [22] is illustrated in Figure 3.

\section{Conclusions}

Both intensity and $\gamma$ can be increased for $\beta$-beams. First studies suggests that a rate of $10^{19}$ neutrinos along one straight section per year of $10^{7}$ seconds could be achievable. The possible upgrade of the CERN SPS injector and the use of the Tevatron as an accelerator [30] for $\beta$-beams could at least allow $\gamma$ 's in the region of 300 for isotopes of interest. The intensity upgrades would beside from important $\mathrm{R} \% \mathrm{D}$ on targets and ion sources, also require a new strategy for magnet protection and accumulation. The higher $\gamma$ would at least for CERN require a new high energy synchrotron (SPS+). Both options will be costly and only detailed studies can answer the question of which option is easier. However, it is clear that at higher $\gamma$ the background problems in the detector appears less severe which would allow for a larger duty factor. This would make it 


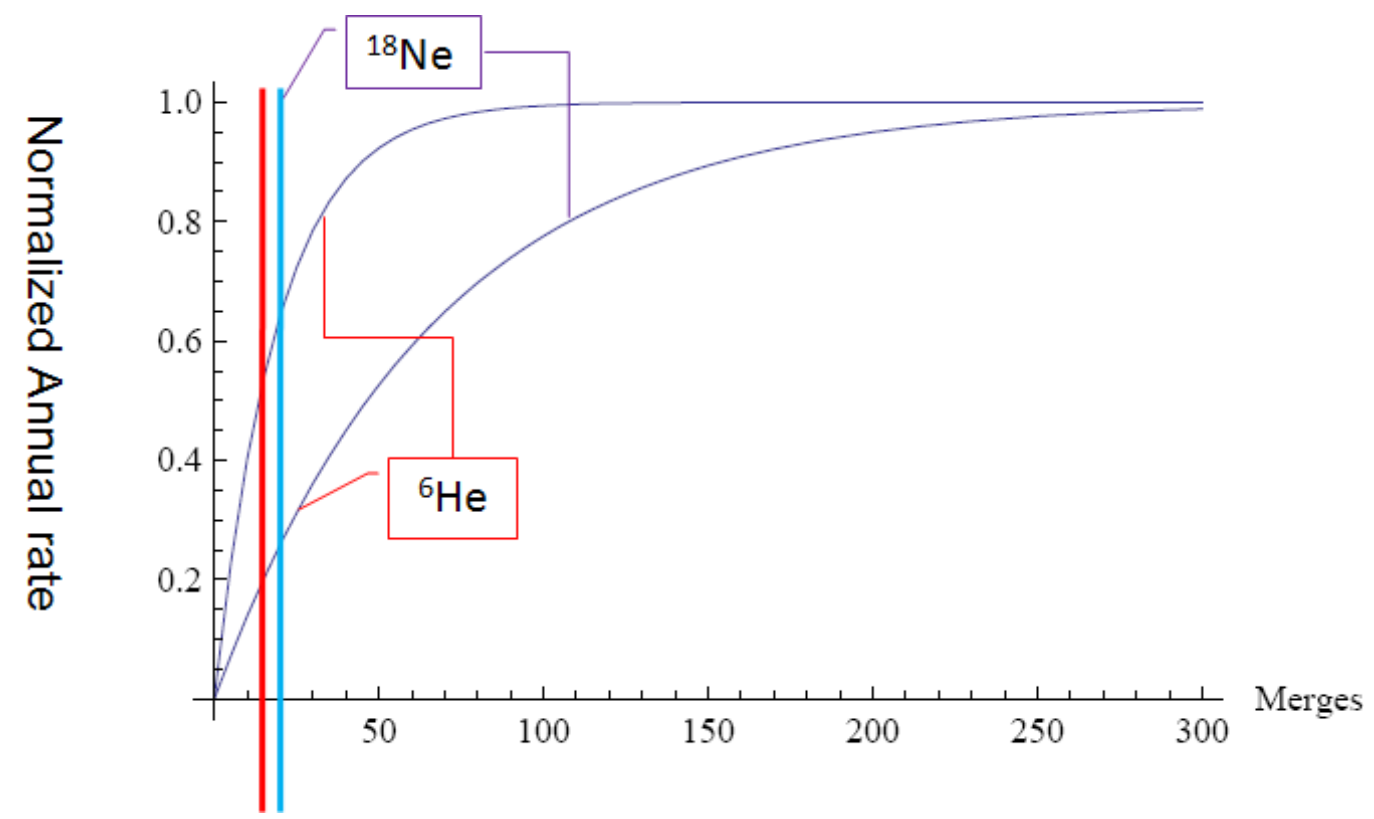

Figure 3: In the diagram the fraction of ${ }^{6} \mathrm{He}$ ions (upper line) and ${ }^{18} \mathrm{Ne}$ ions (lower line) stored in the decay ring using the stacking scheme discussed [22] are plotted as a function of the number of $100 \%$ efficient merges. The ideal case to which the curves eventually converge is the ideal case in which all ions are accumulated in the ring until they decay. The vertical lines for each ion types marks the stacking limits for the $\beta$-beam studied in [22], 15 merges for ${ }^{6} \mathrm{He}$ and 20 merges for ${ }^{18} \mathrm{Ne}$.

possible to use a simpler accumulation scheme which in itself would represent an overall efficiency gain for the facility.

\section{References}

[1] A. Cervera et al., Nucl. Phys. B 579 (2000) 17 [Erratum-ibid. B 593 (2001) 731] [arXiv:hep-ph/0002108]; A. Donini, D. Meloni and P. Migliozzi, Nucl. Phys. B 646 (2002) 321 [arXiv:hep-ph/0206034].

[2] J. Burguet-Castell et al., Nucl. Phys. B 608 (2001) 301 [arXiv:hep-ph/0103258].

[3] H. Minakata and H. Nunokawa, JHEP 0110 (2001) 001 [arXiv:hep-ph/0108085]; G. L. Fogli and E. Lisi, Phys. Rev. D 54 (1996) 3667 [arXiv:hep-ph/9604415]; V. Barger, D. Marfatia and K. Whisnant, Phys. Rev. D 65 (2002) 073023 [arXiv:hep-ph/0112119].

[4] F. Ardellier et al. [Double Chooz Collaboration], arXiv:hep-ex/0606025.

[5] Y. Itow et al., arXiv:hep-ex/0106019.

[6] P. Zucchelli, Phys. Lett. B 532 (2002) 166.

[7] S. Geer, Phys. Rev. D 57 (1998) 6989 [Erratum-ibid. D 59 (1999) 039903] [arXiv:hep-ph/9712290]; A. De Rujula, M. B. Gavela and P. Hernandez, Nucl. Phys. B 547 (1999) 21 [arXiv:hep-ph/9811390].

[8] The ISS Physics Working Group, arXiv:0710.4947 [hep-ph].

[9] J. Burguet-Castell et al., Nucl. Phys. B 695, 217 (2004) [arXiv:hep-ph/0312068].

[10] J. Burguet-Castell et al., Nucl. Phys. B 725, 306 (2005) [arXiv:hep-ph/0503021]. 
[11] A. Donini and E. Fernández-Martínez, Phys. Lett. B 641 (2006) 432 [arXiv:hep-ph/0603261].

[12] C. Rubbia et al., Nucl. Instrum. Meth. A 568 (2006) 475 [arXiv:hep-ph/0602032]; C. Rubbia, arXiv:hep-ph/0609235.

[13] Y. Mori, Development of FFAG accelerators and their applications for intense secondary particle production, Nucl. Instrum. and Methods A 562 (2006) 591; Y. Mori, 39th ICFA Advanced Beam Dynamics Workshop on High Intensity High Brightness Hadron Beams 2006 (HB2006), Tsukuba, Japan, 29 May - 2 Jun 2006.

[14] P. Huber and W. Winter, Phys. Rev. D 68 (2003) 037301 [arXiv:hep-ph/0301257].

[15] S. K. Agarwalla, S. Choubey and A. Raychaudhuri, Nucl. Phys. B 771 (2007) 1 [arXiv:hep-ph/0610333]; S. K. Agarwalla, S. Choubey and A. Raychaudhuri, Nucl. Phys. B 798 (2008) 124 [arXiv:0711.1459 [hep-ph]].

[16] P. Coloma et al., JHEP 0805 (2008) 050 [arXiv:0712.0796 [hep-ph]].

[17] S. K. Agarwalla, S. Choubey and A. Raychaudhuri, Nucl. Phys. B 805 (2008) 305 [arXiv:0804.3007 [hep-ph]].

[18] S. K. Agarwalla et al., JHEP 0806 (2008) 090 [arXiv:0802.3621 [hep-ex]].

[19] C. Volpe, J. Phys. G 34 (2007) R1 [arXiv:hep-ph/0605033].

[20] F. Terranova et al., Eur. Phys. J. C 38 (2004) 69 [arXiv:hep-ph/0405081].

[21] Y. Ashie et al. [Super-Kamiokande Collaboration], Phys. Rev. D 71 (2005) 112005 [arXiv:hep-ex/0501064].

[22] B.Autin et al., The acceleration and storage of radioactive ions for a neutrino factory, CERN/PS 2002-078 (OP), Nufact Note 121, Proceedings of Nufact 02, London, UK, 2002, J. Phys. G: Nucl. Part. Phys. 29 (2003) 1785-1795

[23] M. Benedikt et al., Potential for neutrino and radioactive beam physics of the foreseen upgrades of the CERN accelerators CERN-AB-2006-019-PAF (2006)

[24] M. Benedikt and S. Hancock, A novel scheme for injection and stacking of radioactive ions at high energy, Nucl. Instrum. Meth. A 550 (2005) 1.

[25] M. Blaskiewicz and J.M.Brennan, A Barrier Bucket Experiment for Accumulating De-bunched Beam in the AGS, Proceedings of the European Particle Accelerator Conference, Barcelona, Spain, 1996

[26] M.Loislet and S.Mitrofanov, Alternative production scenarios for ${ }^{6} \mathrm{He}$ and ${ }^{18} \mathrm{Ne}$, Oral presentation at the 6th Beta-beam Task Meeting, EURISOL, 19th November 2007, http://eurisol.org

[27] M.Hass et al. , Light radioisotopes for nuclear astrophysics and neutrino physics, J. Physics G35, 104042 (2008)

[28] F.W. Jones and E. Wildner, Simulation of decays and secondary ion losses in a betabeam decay ring, Proceedings of PAC07, Albuquerque, New Mexico, USA

[29] R. Gupta et al. Open midplane dipole design for LHC IR upgrade, IEEE Trans. Appl. Supercond. 14 (2004) 259.

[30] C. Albright et al., The Neutrino Factory, Muon Collider Collaboration, Neutrino Factory and Beta Beam Experiments and Developments, in APS Joint Study Report on the Future of Neutrino Physics, FNAL-TM-2259, 2004. http://arxiv.org/abs/physics/0411123 